\title{
Convective forces increase rostral delivery of intrathecal radiotracers and antisense oligonucleotides in the cynomolgus monkey nervous system
}

Jenna M. Sullivan ${ }^{2,3 \dagger}$, Curt Mazur ${ }^{1 *+}$ (D), Daniel A. Wolf ${ }^{2}$, Laura Horky $^{2}$, Nicolas Currier ${ }^{2}$, Bethany Fitzsimmons $^{1}$, Jacob Hesterman ${ }^{3}$, Rachel Pauplis ${ }^{3}$, Scott Haller ${ }^{4}$, Berit Powers', Leighla Tayefeh', Bea DeBrosse-Serra', Jack Hoppin ${ }^{3}$, Holly Kordasiewicz ${ }^{1}$, Eric E. Swayze ${ }^{1}$ and Ajay Verma ${ }^{2}$

\begin{abstract}
Background: The intrathecal (IT) dosing route introduces drugs directly into the CSF to bypass the blood-brain barrier and gain direct access to the CNS. We evaluated the use of convective forces acting on the cerebrospinal fluid as a means for increasing rostral delivery of IT dosed radioactive tracer molecules and antisense oligonucleotides (ASO) in the monkey CNS. We also measured the cerebral spinal fluid (CSF) volume in a group of cynomolgus monkeys.

Methods: There are three studies presented, in each of which cynomolgus monkeys were injected into the IT space with radioactive tracer molecules and/or ASO by lumbar puncture in either a low or high volume. The first study used the radioactive tracer ${ }^{64} \mathrm{Cu}$-DOTA and PET imaging to evaluate the effect of the convective forces. The second study combined the injection of the radioactive tracer ${ }^{99 \mathrm{~m}} \mathrm{Tc}$-DTPA and ASO, then used SPECT imaging and ex vivo tissue analysis of the effects of convective forces to bridge between the tracer and the ASO distributions. The third experiment evaluated the effects of different injection volumes on the distribution of an ASO. In the course of performing these studies we also measured the CSF volume in the subject monkeys by Magnetic Resonance Imaging.

Results: It was consistently found that larger bolus dose volumes produced greater rostral distribution along the neuraxis. Thoracic percussive treatment also increased rostral distribution of low volume injections. There was little added benefit on distribution by combining the thoracic percussive treatment with the high-volume injection. The CSF volume of the monkeys was found to be $11.9 \pm 1.6 \mathrm{~cm}^{3}$.
\end{abstract}

Conclusions: These results indicate that increasing convective forces after IT injection increases distribution of molecules up the neuraxis. In particular, the use of high IT injection volumes will be useful to increase rostral CNS distribution of therapeutic ASOs for CNS diseases in the clinic.

Keywords: Intrathecal, Lumbar puncture, Convective forces, Antisense oligonucleotide, PET imaging, SPECT imaging, CT imaging

*Correspondence: cmazur@ionisph.com

†Jenna M. Sullivan and Curt Mazur-co-first authors

${ }^{1}$ Ionis Pharmaceuticals, Inc., 2855 Gazelle Court, Carlsbad, CA 92010, USA

Full list of author information is available at the end of the article

\section{Background}

The development of novel CNS treatment modalities can be hampered by the lack of blood brain barrier (BBB) permeability. The intrathecal (IT) dosing route allows for direct delivery of therapeutic molecules to the central

(c) The Author(s) 2020. This article is licensed under a Creative Commons Attribution 4.0 International License, which permits use, sharing, adaptation, distribution and reproduction in any medium or format, as long as you give appropriate credit to the original author(s) and the source, provide a link to the Creative Commons licence, and indicate if changes were made. The images or other third party material in this article are included in the article's Creative Commons licence, unless indicated otherwise in a credit line to the material. If material is not included in the article's Creative Commons licence and your intended use is not permitted by statutory regulation or exceeds the permitted use, you will need to obtain permission directly from the copyright holder. To view a copy of this licence, visit http://creativeco mmons.org/licenses/by/4.0/. The Creative Commons Public Domain Dedication waiver (http://creativecommons.org/publicdomain/ zero/1.0/) applies to the data made available in this article, unless otherwise stated in a credit line to the data. 
nervous system (CNS), bypassing the blood brain barrier. The most practical routine clinical route for IT drug delivery is via lumbar puncture, which introduces drug into the lumbar CSF cistern at the caudal end of the neuraxis. IT administration has been successfully used to deliver the antisense oligonucleotide (ASO) therapeutic Spinraza to patients with Spinal Muscular Atrophy [1]. Additionally, several other BBB impermeable therapeutic molecules in development, including proteins, nucleic acids, viral gene therapy vectors, stem cells and exosomes are pursuing use of the IT dosing route to target diseases of the CNS. Since these approaches aim to treat various diseases in both pediatric and adult patient populations that can be associated with significant variability in intersubject anatomy and CSF volumes, common principles are needed to optimize the IT dosing procedure for broad neuraxial delivery. Approaches that increase neuraxial drug exposure after lumbar IT delivery will be useful as ASO therapies, and other intrathecally delivered modalities, advance into indications that involve more rostral brain structures.

Broad CNS tissue drug delivery following IT dosing requires neuraxial movement within the subarachnoid CSF followed by entry into the CNS parenchyma [2]. Drug movement within the subarachnoid space is largely facilitated by several CSF convective mechanisms including CSF turnover, cardiac motion, respiratory thoracic motion, and body movement [3-6]. We recently demonstrated in rodent studies that convective force generated in the CSF by the IT dosing procedure itself can be leveraged to increase the spread of drug along the neuraxis [7]. Here, we evaluate the convection enhancing effects of different dosing bolus volumes and externally applied percussive force on the neuraxial distribution and pharmacodynamic effect of ASOs delivered via IT lumbar puncture in cynomolgus monkeys with the goal of enhancing the cranial distribution and widespread CNS action of therapeutic ASOs.

To evaluate the effects of these convection inducing factors on IT drug delivery in a larger species, we conducted a series of experiments in non-human primates following IT delivery of imaging agents and ASOs. First, we quantified neuraxial distribution after IT drug delivery, both with and without the use of convective forces, by PET imaging of the commonly used small molecule non-protein binding imaging agent ${ }^{64}$ Copper-dodecane tetraacetic acid $\left({ }^{64} \mathrm{Cu}\right.$-DOTA),. We then replicated these efforts with an ASO that was co-injected with ${ }^{99 \mathrm{~m}} \mathrm{Tech}$ netium diethylenetriaminepentaacetic acid $\left({ }^{99 \mathrm{~m}} \mathrm{Tc}-\right.$ DTPA) to allow for SPECT imaging to define the early distribution effects of the convective forces. Then the distribution, pharmacokinetics and pharmacodynamics of the ASO were evaluated and compared with the
${ }^{99} \mathrm{~m}$ Tc-DTPA imaging data. Finally, using a therapeutically relevant ASO targeting microtubule associated protein tau (MAPT), with the potential to treat Alzheimer's disease and frontotemporal dementia [8], we confirmed that increasing convective forces can increase rostral CNS delivery of ASOs and imaging agents.

\section{Methods \\ Animals}

Adult cynomolgus monkeys were used in the experiments described herein. Six were used for the ${ }^{64} \mathrm{Cu}$ DOTA experiment, 24 were used for the ${ }^{99 \mathrm{~m}} \mathrm{Tc}$-DTPA/ ASO experiment and 10 were used for the MAPT ASO experiment. The ${ }^{64} \mathrm{Cu}$-DOTA and ${ }^{99 \mathrm{~m}} \mathrm{Tc}-\mathrm{DTPA} / \mathrm{ASO}$ experiments were conducted at MPI Research (Mattawan, MI) and the MAPT ASO experiment was performed at Northern Biomedical Research (NBR, Norton Shores, MI). The six monkeys in the ${ }^{64} \mathrm{Cu}$-DOTA experiment were non-naïve, acquired from an existing colony at MPI Research (Mattawan, MI). The group was divided into three males and three females of $3.8 \pm 0.7 \mathrm{~kg}$ body weight. The 24 animals in the ${ }^{99 \mathrm{~m}} \mathrm{Tc}-\mathrm{DTPA} / \mathrm{ASO}$ experiment were non-naïve and included 13 males and 11 females. Of this cohort, 20 animals received ${ }^{99 \mathrm{~m}} \mathrm{Tc}$ DTPA/ASO and had a body weight of $4.1 \pm 0.5 \mathrm{~kg}$. The remaining four animals were kept naïve to ${ }^{99 \mathrm{~m}} \mathrm{Tc}$-DTPA/ ASO their CNS tissues were used as controls for the determination of metastasis associated lung adenocarcinoma (Malat1) RNA expression levels. For the MAPT experiment 10 animals were used, 5 females and 5 males, their body weights were between 3.0 and $4.5 \mathrm{~kg}$. For all the animals, fluorescent lighting was provided via an automatic timer for $12 \mathrm{~h}$ per day, tap water was supplied ad libitum via an automatic water system and they received an adequate supply of primate chow except during designated fasting periods.

\section{Anesthesia}

Each animal was fasted for 4-12 h prior to sedation with ketamine $(10-25 \mathrm{mg} / \mathrm{kg}$. IM) for transport to the locations where injections and imaging were performed which are outfitted with the equipment for gas anesthesia maintenance. In the injection and imaging locations, animals were intubated and placed on isoflurane (1-2\%) in oxygen carrier gas for the lumbar puncture, percussive wrap treatment when indicated, and imaging when indicated. Vital signs were monitored throughout the procedure. Lactated Ringer's Solution was administered in a $5 \mathrm{~mL}$ subcutaneous (SC) bolus both pre- and postimaging. Each animal was also given intramuscular (IM) Metacam $(0.2 \mathrm{mg} / \mathrm{kg}, \mathrm{IM})$ and Ceftiofur $(2.2 \mathrm{mg} / \mathrm{kg}, \mathrm{IM})$. 


\section{Magnetic Resonance Imaging (MRI)}

Whole body MRI was acquired from each animal to measure CSF volume. All animals were imaged on a $1.5 \mathrm{~T}$ Siemens Symphony MRI scanner (Siemens Medical Systems, Erlangen, Germany). High-resolution MRI of the spine and cranium were acquired in separate acquisitions within a single imaging session while each animal was under anesthesia. Images were acquired in axial orientation using a 3D T2 fast-spin echo (FSE) sequence with one excitation. Cranium data were acquired with a clinical CP extremity coil, voxel size of $0.7 \times 0.7 \times 2 \mathrm{~mm}$, acquisition time of $8.05 \mathrm{~min}$, TR of $12.5 \mathrm{~ms}$, and TE of $6.25 \mathrm{~ms}$. Body data were acquired with a clinical body coil, voxel size of $0.35 \times 0.35 \times 2 \mathrm{~mm}$, acquisition time of $13.97 \mathrm{~min}$, relaxation time of $11.82 \mathrm{~ms}$, and excitation time of $5.91 \mathrm{~ms}$.

\section{MRI data analysis}

The cranium and body MRI data were stitched, including alignment, co-registration, and intensity blending in overlapping slices. Coarse, manually defined regions-ofinterest (ROIs) were used to segment the brain and spine, including nearby surrounding tissue. Sub-regions with relative homogeneous CSF intensity within the brain and coarse spine ROI regions were selected. Sub-region-specific intensity thresholds were used to segment the final CSF ROI. In sub-regions with insufficient contrast, the CSF was segmented manually using MRI intensity as a guide.

\section{Lumbar puncture procedure}

After each animal was anesthetized, an experienced veterinary surgeon injected the radiotracer and/or ASO into the intrathecal space by lumbar puncture. In the experiments performed at MPI, the animals were placed in left lateral recumbence and a 22-gauge Quinke spinal needle was introduced into the L4/L5 intrathecal space using aseptic technique. In the MAPT experiment performed at NBR, the animals were placed vertically in a seated position and the torso extended over a circular form. A 25 -gauge Pencan Paed ${ }^{\circledR}$ pencil-point needle for pediatric use (B Braun) was inserted into the L4/L5 intervertebral space and used to inject the dosing solution. In all cases, the placement of the needle was verified by the presence of CSF at the needle hub pre- and post-injection. Once placement of the needle was verified, the radiotracer or ASO was injected over $\sim 2 \mathrm{~min} / \mathrm{mL}$ volume, and then the needle was withdrawn.

Percussive wrap and high frequency chest wall oscillation In the ${ }^{64} \mathrm{Cu}$-DOTA and ${ }^{99 \mathrm{~m}} \mathrm{Tc}$-DTPA/ASO experiments, a percussive wrap that delivers high-frequency chest wall oscillation (HFCWO) therapy (SmartVest ${ }^{\circledR}$, Electromed, Inc., New Prague, MN) was applied to each animal after IT injection. The SmartVest ${ }^{\circledR}$ system is an airway clearance system prescribed for patients with compromised airway clearance (such as in cystic fibrosis or spinal muscular atrophy). The wearable wrap consists of an inflatable bladder connected to an air pulse generating system. A size "S-Small" Single-Patient Use SmartVest Wrap ${ }^{\circledR}$ with wrap height of $10.5 \mathrm{~cm}\left(41 / 4^{\prime \prime}\right)$ was used on all monkeys. Treatments were conducted at $5 \mathrm{~Hz}$ and $10 \mathrm{psi}$. Under percussive vest treatment conditions, animals were anaesthetized, the IT injections performed, then the wrap was applied to each animal's torso. HFCWO treatment was applied for 30 min where indicated, otherwise the animal remained in the wrap for $30 \mathrm{~min}$ without activation of the air pulse mechanism. Following percussive wrap treatment, the wrap was removed, and each animal was placed in the PET or SPECT scanner for imaging.

\section{${ }^{64} \mathrm{Cu}$-DOTA radiochemistry}

DOTA chelator was purchased from Macrocyclics (Lot M14010001-070713). ${ }^{64} \mathrm{Cu}$ was supplied in $0.1 \mathrm{M} \mathrm{HCl}$ by Washington University (St. Louis, MO). ${ }^{64} \mathrm{Cu}$-DOTA was prepared prior to each imaging session. Briefly, $0.1 \mathrm{M}$, $\mathrm{pH} 6$ citrate buffer, and ${ }^{64} \mathrm{Cu}$ were added to a glass vial. Following the addition of radioactivity, chelator solution $(1.0 \mathrm{mg} / \mathrm{mL}$ in $0.1 \mathrm{M}$ citrate buffer, $\mathrm{pH} 6)$ was added to the vial. The vial was vortexed for 1 min then placed in a water bath at $50{ }^{\circ} \mathrm{C}$ for $30 \mathrm{~min}$. Following chelation complex formation, two instant thin layer chromatography (ITLC) runs were conducted in parallel to confirm chelation efficiency.

For ITLC, a small aliquot of the ${ }^{64} \mathrm{Cu}$-chelator solution was diluted 1:100 in $200 \mu \mathrm{L} .2 \mu \mathrm{L}$ were drawn up by pipette and dispensed onto the bottom of the ITLC paper strip and allowed to dry. Once dry, the ITLC strip was placed in a $15 \mathrm{~mL}$ conical tube containing $800 \mu \mathrm{L}$ of ITLC developing buffer (0.1 M citrate buffer, $\mathrm{pH}$ 6) and chelation efficiency was determined. Chelation efficiency was consistently $>99 \%$. Specific activity (SA) was $358.9 \pm 166.5 \mathrm{MBq} / \mathrm{mg}$ (mean $\pm \mathrm{SD}, \mathrm{n}=8$ runs).

\section{${ }^{64}$ Cu-DOTA study design}

The tissue distribution of ${ }^{64} \mathrm{Cu}$-DOTA radioactivity was evaluated in 6 cynomolgus monkeys after IT bolus delivery of $\sim 18 \mathrm{MBq}{ }^{64} \mathrm{Cu}$-DOTA under 4 conditions. Each condition consisted of an IT delivery of ${ }^{64} \mathrm{Cu}$-DOTA followed by dynamic, whole-body PET imaging. All animals were imaged under each condition in a crossover study design with 1-2 weeks between conditions. The tests on the different experimental days were low $(0.36 \mathrm{~mL})$ versus high $(1.8 \mathrm{~mL})$ injection volume, low injection volume versus low injection volume with 30 -min percussive wrap 
treatment, and high injection volume versus high injection volume with 30 -min percussive wrap treatment. There were no significant differences in molar mass of ${ }^{64} \mathrm{Cu}$-DOTA injected between conditions. Following the experiments, the animals were returned to the MPI colony.

\section{PET image acquisition}

Whole-body continuous bed motion positron emission tomography (PET) and computed tomography (CT) data were acquired on a Focus220 microPET (Siemens Medical Systems, Knoxville, TN) and CereTom CT (NeuroLogica Corp, Danvers, MA), respectively. All animals were imaged in the head-first prone position. Radioactive fiducial markers were placed on the bed at three different positions for each scan to support coregistration of the PET time frames and as a standard of radioactivity. Dynamic PET data were acquired for either 0-120 min in scans without percussive treatment or 30-120 min in scans with percussive treatment PET data were acquired in 3D list-mode and re-binned into 2D sinograms. PET images were reconstructed by a 2D Ordered Subset Expectation Maximization (OSEM2D) algorithm with 14 subsets and 4 iterations. Corrections were made for detector normalization, decay, dead-time, random coincidences, and attenuation into images with $256 \times 256 \times 693$ pixels and $0.95 \times 0.95 \times 0.80 \mathrm{~mm}$ voxels. Co-registered whole-body CT data, segmented based on Hounsfield units into bone, tissue, and lung compartments, were used for attenuation correction.

After each PET scan, a CT was acquired for anatomical registration. All animals were imaged on a specially design bed, which was transferred from the PET to the $\mathrm{CT}$ to allow consistent positioning of the animal between the two modalities. CT acquisition time was $6 \mathrm{~min}$ and based on an axial range of $450 \mathrm{~mm}$, a tube peak voltage of $120 \mathrm{kVp}$, with 720 projections per rotation, and $4 \mathrm{~s}$ per projection.

\section{Estimation of tissue uptake of ${ }^{64} \mathrm{Cu}$-DOTA}

ROIs were defined for the cranium, heart, liver, and kidneys by fitting ellipsoids of fixed volume to each organ based on CT. The cranium ROI contained both parenchyma and CSF within and surrounding the brain. The bladder ROI was defined through automated thresholding of the PET signal in the bladder or drawn by hand based on anatomy (in cases of low bladder signal). Spinal column ROIs were defined by applying a combination of manual and automated segmentation thresholds to the CT. The CSF ROI included both the spinal cord and CSF within the IT space. The CSF ROI was divided into cervical, thoracic, and lumbar sub-regions based on vertebral level.
Quantitative data from these ROIs were extracted and radioactivity concentration in units of percent injected dose per gram (\%ID/g) at each time point for each ROI was plotted (assuming $1 \mathrm{~cm}^{3}$ is equivalent to $1 \mathrm{~g}$ of tissue). The area-under-the-curve (AUC) was calculated for each ROI. For AUC comparisons from conditions with and without percussive wrap treatment, AUCs for all regions were calculated from 30 to $120 \mathrm{~min}$ of data post-injection. Plots of radioactivity concentration (\%ID/g) over the length of the spine were also generated for each ROI. All analyses were performed in VivoQuant ${ }^{\mathrm{TM}}$ 1.23patch1-3 and MATLAB R2014a (MathWorks ${ }^{\circledR}$, Natick, MA, USA).

\section{${ }^{99 m}$ Tc-DTPA SPECT/ASO study design}

Twenty cynomolgus monkeys were randomly assigned to 4 treatment groups of 5 each. All treatment groups received $16 \mathrm{mg}$ of the ASO against MALAT1 co-injected into the lumbar IT space with $\sim 37 \mathrm{MBq}$ of ${ }^{99 \mathrm{~m}} \mathrm{Tc}$-DTPA. Two treatment groups received the $\mathrm{ASO} /$ tracer injection in a volume of $0.8 \mathrm{~mL}$. These two groups were then divided into one with and one without $30 \mathrm{~min}$ of percussive wrap treatment. The other two treatment groups received the $\mathrm{ASO} /$ tracer injection in a volume of $2.4 \mathrm{~mL}$. These two groups were also divided into one with and one without $30 \mathrm{~min}$ of percussive wrap treatment. An additional 4 animals naïve to any treatment were used as negative controls for the RNA expression and ASO concentration determinations.

\section{Antisense oligonucleotide}

The antisense oligonucleotide against both MALAT1 and $M A P T$ were developed by in vitro and in vivo screening techniques at Ionis Pharmaceuticals and were shown to be cross reactive to cynomolgus monkey (data not shown). These ASOs are both 20 base long chemically modified DNA molecules. The MALAT1 ASO has the sequence GCCAGGCTGGTTATGACTCA and the MAPT ASO the sequence ACACACCTTCATTTACTG TC. In both of the ASOs the 5 bases on both the $5^{\prime}$ and $3^{\prime}$ ends have $2^{\prime}$ methoxyethyl (MOE) sugar modifications and the backbones are mixtures of phosphorothioate and phosphodiester linkages. Both ASOs were formulated in sterile artificial cerebral spinal fluid (aCSF). The Malat1 ASO was formulated at 20 and $6.7 \mathrm{mg} / \mathrm{mL}$ for doses of $16 \mathrm{mg}$ in 0.8 and $2.4 \mathrm{~mL}$, respectively. The MAPT ASO was formulated at 50 and $20 \mathrm{mg} / \mathrm{mL}$ for doses of $40 \mathrm{mg}$ in 0.8 and $2.0 \mathrm{~mL}$, respectively. Both ASOs were sterile filtered through a $0.22 \mu \mathrm{m}$ filter and placed in sterile septum vials which were crimped closed. The ASO vials were sent to the experimental sites for injection of the ASO into the animals. 


\section{SPECT image acquisition}

Immediately after IT ${ }^{99 m}$ Tc-DTPA delivery, the animals were maintained under anesthesia and SPECT data were acquired using a four-headed SPECT/CT scanner (NanoSPECT/CT, Bioscan, Inc.) for $10 \mathrm{~min}$ (time 0 scan). Following this scan, each animal was placed in the percussive wrap for a 30 min percussive wrap treatment (either activated or not) then imaged again with the SPECT scanner for $30 \mathrm{~min}$ (time $40 \mathrm{~min}$ scan). The animals were allowed to recover from the anesthesia and then were re-anesthetized for a final $30 \mathrm{~min}$ SPECT scan at $6 \mathrm{~h}$ post IT injection. The animals were again allowed to recover from anesthesia. All SPECT scans were followed immediately by a CT scan.

SPECT projection data were acquired head to mid torso using a UHR parallel-hole collimator with an energy window of 126.5-154.6 keV, 96 total projections and $25 / 75 \mathrm{~s}$ per projection for $10 / 30$ min scans, respectively. SPECT projection images were reconstructed in the software ReSPECT v 2.5 (SciVis, Germany) using a maximum likelihood estimation method (MLEM) with 6 iterations. A user-defined threshold was chosen to define the body contour and a fixed attenuation coefficient of $0.12 / \mathrm{cm}$ was utilized for attenuation correction. No smoothing was used in reconstruction and data were reconstructed into $1 \mathrm{~mm}$ isotropic voxels.

CT scans were performed from head to mid torso region on a CereTom CT (NeuroLogica Corp, Danvers, MA). Acquisition time was $604 \mathrm{~s}$ with tube peak voltage of $120 \mathrm{kVp}$, current set to $4 \mathrm{~mA}, 288$ projections per rotation, and $6 \mathrm{~s}$ per projection.

\section{SPECT image processing and image generation}

A single quantification factor (QF) was calculated and applied to each SPECT image. Three fiducial markers, consisting of vials containing a known amount of activity were included in each SPECT scan. Each fiducial marker was manually segmented from the unprocessed reconstructed SPECT image, and the given activity in that fiducial marker (decay-corrected to the scan time) was divided by the counts from the segmentation to obtain a QF for that fiducial. The QFs for the three fiducials were then averaged to obtain the QF for the image.

SPECT and CT images at each time point were coregistered. Maximum intensity projection (MIP) images of co-registered SPECT and CT were generated with SPECT data scaled from 0 to $1 \% \mathrm{ID} / \mathrm{g}$. Image processing and MIP generation was performed in VivoQuant 2.0. Full quantitative analysis of the image data was not performed.

\section{${ }^{99 m}$ Tc-DTPA/MALAT1 ASO experiment-euthanasia and tissue harvesting}

One week (7 days) following the IT dosing procedure in the ${ }^{99 \mathrm{~m}}$ Tc-DTPA/MALAT1 ASO experiment, the animals were euthanized by injection of euthanasia solution (EUTHASOL-pentobarbital sodium and phenytoin sodium solution, Virbac AH, Inc). Euthanasia was confirmed by the secondary method of exsanguination via femoral vessel incision. The brain, and spinal cord were harvested from the animals. The brains were placed in a monkey brain matrix (ASI Instruments, MBM-2500C) and sliced into $4 \mathrm{~mm}$ thick coronal slabs. Each of these slabs was divided into left and right hemispheres. The left hemisphere was immersion fixed in $10 \%$ phosphate buffered formaldehyde solution and the right hemisphere was frozen between Parafilm sheets on an aluminum plate cooled with dry ice and the tissue was buried under powdered dry ice. The spinal cord was divided into cervical, thoracic and lumbar sections from which small caudal pieces of the sections were immersion fixed in 10\% phosphate buffered formaldehyde solution and the rostral pieces buried in powdered dry ice to freeze. All frozen tissues were shipped to Ionis Pharmaceuticals for analysis of MALAT1 RNA and tissue concentrations of the MALAT1 ASO. The fixed tissues were embedded in paraffin and histological sections were taken for immunohistochemistry (IHC) of the ASO and in situ staining of MALAT1 RNA.

\section{MALAT1 RNA PCR analysis}

Tissue punches $\left(\sim 12 \mathrm{~mm}^{3}\right)$ were taken from fresh frozen brain and spinal cord slices and homogenized with sterile ceramic beads in guanidinium thiocyanate buffer containing $8 \% 2$-mercaptoethanol using a bead homogenizer. Total RNA was prepared from tissue lysates using a RNeasy 96 kit (Qiagen). The prepared RNA was assayed for MALAT1 and cyclophilin A levels using primer/TaqMan probe sets with an EXPRESS One-Step SuperScript quantitative reverse transcriptase polymerase chain reaction (qRT-PCR) kit (Invitrogen). qRTPCR plates were run on StepOnePlus Real-Time PCR machines (Applied Biosystems) and data was initially analyzed using StepOne Software (Applied Biosystems). MALAT1 RNA expression level was normalized to cyclophilin $A$ mRNA expression to correct for the amount of RNA in the reaction. Malat1 RNA expression level was further normalized as percent naïve control.

Malat1 primers/probe sequences:

Forward 5'-AAAGCAAGGTCTCCCCACAA-3', Reverse 5'-GTGAAGGGTCTGTGCTAGATC-3', 
Probe 5'-/56-FAM/CAACTTCTC/ZEN/TGCCAC ATCGCCACCT/3IABkFQ-3';

\author{
Cyclophilin A primers/probe sequences:
}

Forward 5'-CGACGGCGAGCCTTTG-3', Reverse $5^{\prime}$-TCTGCTGTCTTTGGAACCTTGTC-3', Probe 5'-/56-FAM/CGCGTCTCCTTCGAGCTG TTTGC/36-TAMSp/-3'.

\section{Analysis of tissue levels of the MALAT1 and MAPT ASOs}

Frozen tissue samples from frontal cortex, lumbar, thoracic and cervical spinal cord were obtained from the frozen monkey tissues. Where possible the samples for PCR analysis and tissue concentrations of the Malat1 ASO were adjacent samples. The samples were minced, weighed into individual wells in a 96-well plate, and had $500 \mu \mathrm{L}$ homogenization buffer added to those wells. Control tissue homogenate for curves was made by weighing control monkey brain and adding homogenization buffer at a 9 to 1 ratio. Five hundred microliter aliquots were pipetted into a 96-well plate and appropriate amounts of calibration standards were spiked in the corresponding wells. Wells containing samples and calibration standards then had internal standard (IS) and approximately $0.25 \mathrm{~cm}^{3}$ granite beads added. The plates were then extracted via a liquid-liquid extraction with ammonium hydroxide and phenol:chloroform:isoamyl alcohol (25:24:1). The aqueous layer was then further processed via solid phase extraction on a Strata X plate (Phenomonex Inc., CA). Eluates had a final pass through a protein precipitation plate before they were dried under nitrogen at $50{ }^{\circ} \mathrm{C}$. Dried samples were reconstituted in $140 \mu \mathrm{L}$ water containing $100 \mu \mathrm{M}$ EDTA. An Agilent liquid chromatograph with mass spectrometry detection (LC-MS/ MS) instrument consisting of a 1290 binary pump, a column oven, an auto sampler, and a 6460 triple quadrupole mass spectrometer was used for analysis (Agilent, Wilmington, DE, USA).

The tissue extracts were injected onto a Kinetex analytical column (Phenomenex, $100 \times 2.1 \mathrm{~mm}, 2.6 \mu \mathrm{m}$ particle size; $100 \AA$ ) which was equilibrated with $15 \%$ methanol in $5 \mathrm{mM}$ triethyalamine (TEA) and $400 \mathrm{mM}$ hexo-fluoroisopropanol (HFIP) and then maintained at $55{ }^{\circ} \mathrm{C}$ and with a flow rate of $0.3 \mathrm{~mL} / \mathrm{min}$ throughout the analysis. A gradient from 15 to $50 \%$ methanol over $7 \mathrm{~min}$, increased to $80 \%$ over $0.5 \mathrm{~min}$, and maintained at $80 \%$ for $0.5 \mathrm{~min}$ was used to separate the MALAT1 ASO and internal standard (IS) from background peaks. A re-equilibration time of 2 min at the starting conditions was used between samples.
All mass measurements were made on-line with the scan time set from 2 to $8 \mathrm{~min}$. During that time window, the mass spectrometer was set to scan for MRM transitions for the full length ASO and IS $(-8 \mathrm{~m} / \mathrm{z}$, 893.1-> 94.8; and $-4 \mathrm{~m} / \mathrm{z}, 1160.7->94.8$, respectively). Mass spectra were obtained using a spray voltage of $-1500 \mathrm{~V}$, a nebulizer gas flow of $25 \mathrm{psig}$, a sheath gas flow rate of $12 \mathrm{~L} / \mathrm{min}$ at $350{ }^{\circ} \mathrm{C}$, a drying gas flow rate of $5 \mathrm{~L} / \mathrm{min}$ at $250{ }^{\circ} \mathrm{C}$, and a capillary voltage of $-3750 \mathrm{~V}$. Chromatograms were analyzed using Agilent Mass Hunter software.

Tissue calibration curves were constructed using peak area ratios of the ASO to the IS and applying a weighted $(1 / x)$ Quadratic regression. All tissues had a calibration range for the ASO from 0.036 to $178.82 \mu \mathrm{g} / \mathrm{g}$ in $50 \mathrm{mg}$ monkey brain homogenate. A minimum signal-to-noise ratio of 5:1 was used to distinguish ASO peaks from background. Acceptance criteria for the calibration curve were set to $85-115 \%$ of nominal values. All samples were stored at $-70{ }^{\circ} \mathrm{C} \pm 10{ }^{\circ} \mathrm{C}$, upon receipt. The MAPT ASO was quantified from the tissues using the same method.

\section{In situ staining of MALAT1 RNA and IHC for MALAT1 ASO}

Based on the PCR results, it was determined that animal numbers 1011 and 1006 were typical of the MALAT1 knock down level of the successfully dosed animals in the high volume no wrap and low volume no wrap groups, respectively and were chosen for in situ staining for MALAT1 RNA. Naïve animal 2601 was chosen as a normal control for the MALAT1 in situ staining as comparator. The paraffin embedded tissues were sectioned at $4 \mu \mathrm{m}$ thickness and in the case of the tissue block being larger than the $1^{\prime \prime} \times 3^{\prime \prime}$ slides they were sliced down the center with a razor blade and the two halves were collected onto separate slides. Adjacent sections were taken from all tissue blocks from animals 1011, 1006 and 2601 for the MALAT1 in situ stain and immunohistochemistry for the ASO.

The in situ stain for MALAT1 RNA was performed on a Leica Bond RX research staining robot with a RNAScope custom reagent kit from Advanced Cell Diagnostics (ACD). A 2.5 LS reagent kit-Brown (ACD, \#322100), a RM-Malat1 probe 01-04 (ACD, \#460238), DapB negative control and PPIB positive control were employed for the stain which showed the desired staining results (data not shown). Initially, the slides were dried overnight and then again for $30 \mathrm{~min}$ in a $60{ }^{\circ} \mathrm{C}$ oven. The slides were then loaded into the Bond RX machine and the following RNAScope 2.5 LS reagents were used in the automatic staining procedure: hydrogen peroxide, protease III, AMP1-AMP4, AMP5 and AMP 6 Brown, Rinse, bluing reagent, MALAT1 target probe, Mock probe wash and Leica polymer refine 
detection kit. The automated run was completed then the slides were counterstained with hematoxylin, rinsed in distilled water, dehydrated through graded alcohol to a clearing agent, and cover slipped with Permount (Leica Micromount, \#3801731). The slides were allowed to dry and then were scanned into a Leica Aperio scanner at $20 \times$ resolution.

For the immunohistochemical staining for the ASO, sections were cut from the paraffin embedded blocks at $4 \mu \mathrm{m}$ thickness onto positive charged slides and dried overnight at $50{ }^{\circ} \mathrm{C}$. The tissues were deparaffinized to distilled water and the antigen retrieval performed with Proteinase K (Dako \#S302030-2) treatment for $2 \mathrm{~min}$. Endogenous peroxidase was quenched by incubation in Dako Dual Endogenous Enzyme Blocking Reagent (\#S2003) for 10 min followed with a 30 min incubation in Cyto-Q Background Buster (Innovex, \#NB306-125 ml) to block nonspecific protein binding. The slides were incubated with the custom made anti-ASO primary antibody, 6651 (Pan ASO, Ionis Pharmaceuticals) diluted 1:40,000 for $1 \mathrm{~h}$ at room temperature. The slides were incubated for $30 \mathrm{~min}$ in a 1:200 dilution of the goat anti-rabbit secondary antibody conjugated to horse radish peroxidase (Jackson ImmunoResearch \#111-036-003). The brown color was produced by reaction with 3,3'-diaminobenzidine chromogen (DAB) (Dako, \#K3468) for 5 min and the tissues were counterstained with hematoxylin, dehydrated, cleared and cover slipped with Leica Micromount. After drying, the slides were scanned into the Hamamatsu Nanozoomer scanner at $20 \times$ resolution.

The scanned slides were visualized on a computer and images from the different CNS anatomical regions were acquired by screen capture.

\section{MAPT ASO experimental design}

A group of 7 cynomolgus monkeys was randomly assigned to two groups of 5 for ASO treatment and one group of 2 for vehicle treatment. Each of the two groups of 5 were treated by intrathecal lumbar puncture with $40 \mathrm{mg}$ of the MAPT ASO in either a low $(0.8 \mathrm{~mL})$ or high $(2 \mathrm{~mL})$ dosing volume every 2 weeks for 4 doses (on days $1,14,28$ and 56). The vehicle treated animals were treated with $2 \mathrm{~mL}$ of aCSF on the same dosing schedule. All doses were delivered by hand at an approximate rate of $2 \mathrm{~mL} / \mathrm{min}$. The animals were euthanized 7 days following the final dose (on day 63), brain and spinal cord were harvested and frozen for analysis of MAPT mRNA expression and ASO tissue concentration. Samples of the lumbar spinal cord, thoracic spinal cord, and frontal cortex, were dissected from the frozen tissue samples for MAPT mRNA expression analysis by qRT-PCR and adjacent samples were collected for determination of MAPT ASO concentrations.

\section{Statistics}

In the ${ }^{64} \mathrm{Cu}$-DOTA experiment area under the curve calculations for radioactivity versus time were performed using the trapezoidal rule using GraphPad Prism (version 8.0.2). Comparisons between treatment modalities (high versus low volume, low volume versus low volume with wrap treatment, high volume versus high volume with wrap treatment) were done with two-tailed Welch's T-tests using GraphPad Prism (version 8.0.2). In the ${ }^{99 \mathrm{~m}}$ Tc-DTPA/ASO experiment differences between treatment groups in each tissue for MALAT1 ASO concentration and MALAT1 RNA expression were evaluated with Two-way ANOVAs with Tukey's multiple comparisons test using GraphPad Prism (version 8.0.2). In the MAPT ASO experiment differences between low and high dose volume for MAPT ASO concentrations and MAPT RNA expression were done with two-tailed Welch's T-tests using GraphPad Prism (version 8.0.2). When errors are reported they are standard deviations. For all comparisons, $\mathrm{P}<0.05$ was considered statistically significant. The coefficient of variation was calculated by the formula: Standard deviation/mean $* 100 \%$.

\section{Results}

MRI determination of CSF volume in cynomolgus monkeys

To support the selection of IT dose volumes, total CSF volumes were measured in cynomolgus monkeys. The CSF volume in 20 animals (10 males and 10 females, $3.6 \pm 0.4 \mathrm{~kg}$ body weight, no significant differences between male and female body weights), was $11.6 \pm 1.5 \mathrm{~cm}^{3}$ for the entire neuraxis including the ventricles (Table 1). Total CSF volume was $12.1 \pm 1.6 \mathrm{~cm}^{3}$ in males and $11.0 \pm 1.1 \mathrm{~cm}^{3}$ in females (Table 1). There were no statistically significant differences between males and females in CSF volume (Table 1).

To aid in translation of this work, we chose the experimental dosing volumes in NHP to be a similar percentage of total CSF volume as dose volumes used previously in human patients. High intrathecal dose volumes used in humans have corresponded to approximately $14 \%$ of total adult human CSF volume and volumes of up to $33 \%$ of the total CSF volume have previously been given safely in humans [9-11]. We therefore evaluated the effect of IT dose volumes ranging from $\sim 3$ to $20 \%$ of total monkey CSF volume determined by MRI with the amount of injected molecule kept constant within an experiment. Specifically, IT bolus injection volumes representing $\sim 3 \%(0.36 \mathrm{~mL})$ and $15 \%(1.8 \mathrm{~mL})$ of the total CSF volume were used in the ${ }^{64} \mathrm{Cu}$-DOTA experiment. For the 
Table 1 Whole body CSF volume determined in cynomolgus monkeys using MRI image analysis

\begin{tabular}{|c|c|c|c|}
\hline Animal \# & Sex & Body weight (kg) & $\begin{array}{l}\text { Total CSF } \\
\text { volume } \\
\left(\mathrm{cm}^{3}\right)\end{array}$ \\
\hline A1001 & Male & 3.7 & 14.4 \\
\hline A1002 & Female & 3.1 & 8.8 \\
\hline A1003 & Male & 3.3 & 11.0 \\
\hline A1004 & Female & 3.3 & 10.0 \\
\hline A1005 & Male & 3.7 & 10.3 \\
\hline A1006 & Female & 4.1 & 11.8 \\
\hline A1007 & Female & 3.8 & 11.9 \\
\hline A1008 & Male & 4.2 & 11.7 \\
\hline A1009 & Female & 3.1 & 12.6 \\
\hline A1010 & Male & 3.4 & 12.4 \\
\hline A1011 & Female & 2.8 & 10.4 \\
\hline A1012 & Male & 3.7 & 13.2 \\
\hline A1013 & Female & 3.2 & 10.6 \\
\hline A1014 & Male & 3.1 & 10.4 \\
\hline A1015 & Female & 4.0 & 12.3 \\
\hline A1016 & Male & 4.2 & 11.1 \\
\hline A1017 & Female & 4.1 & 10.9 \\
\hline A1018 & Male & 3.2 & 11.5 \\
\hline A1019 & Male & 4.3 & 14.9 \\
\hline A1020 & Female & 3.4 & 11.0 \\
\hline \multicolumn{4}{|c|}{ Analysis of total CSF volume $\left(\mathrm{cm}^{3}\right)$} \\
\hline Parameter & All animals & Female & Male \\
\hline Mean & 11.6 & 11.0 & 12.1 \\
\hline SD & 1.5 & 1.1 & 1.6 \\
\hline CV\% & $12.7 \%$ & $10.4 \%$ & $13.3 \%$ \\
\hline Median & 11.3 & 10.9 & 11.6 \\
\hline Max & 14.9 & 12.6 & 14.9 \\
\hline Min & 8.8 & 8.8 & 10.3 \\
\hline Range & $8.8-14.9$ & $8.8-12.6$ & $10.3-14.9$ \\
\hline $\begin{array}{l}\text { Female vs. male } \\
\text { T-test } P \text { value }\end{array}$ & & 0.1043 & \\
\hline
\end{tabular}

${ }^{99 \mathrm{~m}}$ Tc-DTPA/Malat1 ASO experiment, IT bolus injection volumes representing 7\% $(0.8 \mathrm{~mL})$ and $20 \%(2.4 \mathrm{~mL})$ of total CSF volume were injected, and for the MAPT ASO experiment IT bolus volumes representing 7\% $(0.8 \mathrm{~mL})$ or high injection volume representing $\sim 17 \%(2 \mathrm{~mL})$ of total CSF volume were injected.

\section{Exclusion of animals from data analysis}

In some cases, in the ${ }^{64} \mathrm{Cu}$-DOTA and the ${ }^{99 \mathrm{~m}} \mathrm{Tc}-\mathrm{DTPA} /$ ASO experiments it was determined that there were IT doses missed due to technical failures. These animals were removed from further analysis. The following are the criteria used for the exclusion of these animals.
In the ${ }^{64} \mathrm{Cu}$-DOTA experiment, the surgeon assessed all IT injections to be successful, except for the injection for one subject under the low volume plus wrap condition. Despite the apparent success of the injections, the image data suggested that at least some injections were multi-compartmental (e.g. IT and epidural space) or only partially in the IT space (Fig. 1a). A criterion to exclude poor or partial IT-injections was defined based on image features: images in which there was an obvious subdermal injection, a non-continuous signal in the IT space or a strong kidney signal at the first imaging time point after injection (reflecting early leakage to the periphery) were excluded from subsequent analyses. Six scans were excluded from analysis 

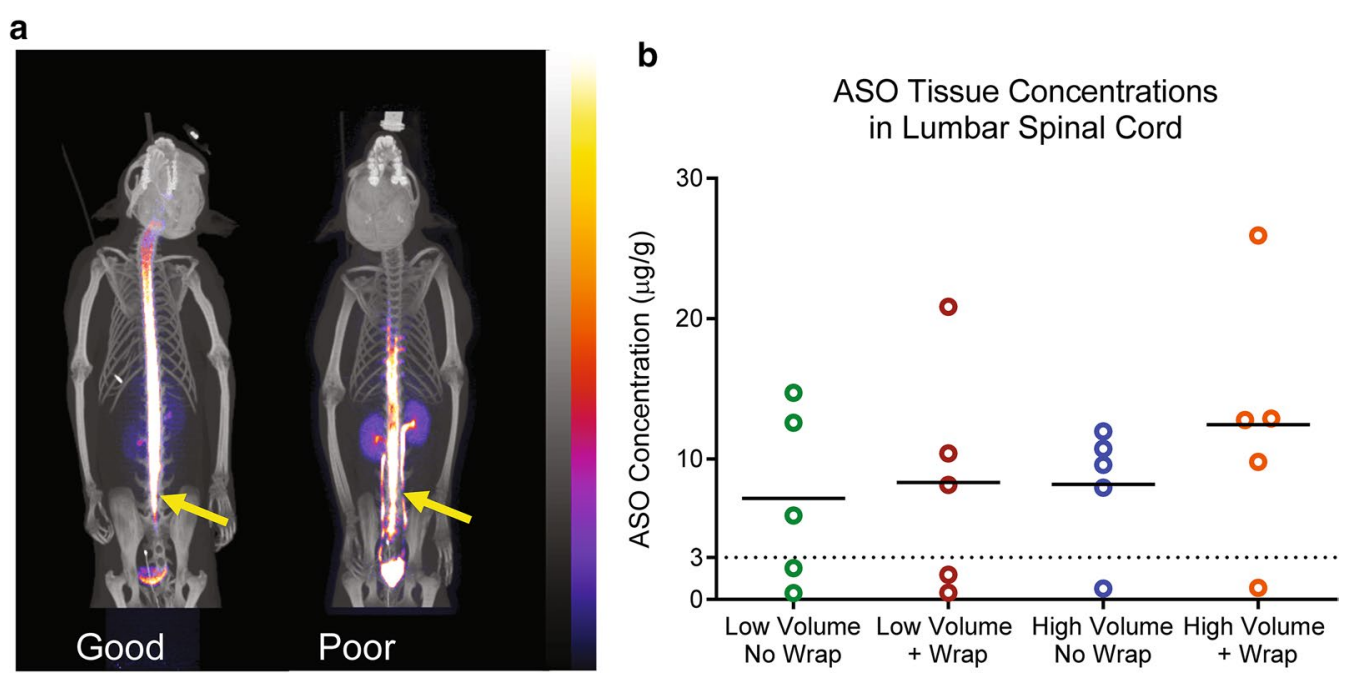

Fig. 1 a Imaging rejection criteria for poor technical injections in the ${ }^{64} \mathrm{Cu}$-DOTA experiment. The PET/CT scan on the left is indicative of a successful IT injection where the radioisotope is mostly contained in the neuraxial compartments. The PET/CT scan on the right illustrates an unsuccessful IT injection where a high radioisotope signal can be seen in the kidneys, ureters and bladder. Sites of injection of the ${ }^{64} \mathrm{Cu}-\mathrm{DOTA}$ are indicated by arrows. PET images were smoothed with a $2.5 \mathrm{~mm}$ Gaussian filter and scaled from 0 to 2\% ID/g indicated by the color scale bar. b Graph of the ASO tissue concentrations in $\mu \mathrm{g} / \mathrm{g}$ tissue in the lumbar spinal cords of all the animals. Animals with lumbar spinal cord tissue concentrations below $3 \mathrm{\mu g} / \mathrm{g}$ tissue were considered probable missed injections due to technical reasons. There were two animals in both low volume $(0.8 \mathrm{~mL})$ groups and one animal in each high dose volume $(2.4 \mathrm{~mL})$ group that had lumbar spinal cord concentrations below $3 \mu \mathrm{g} / \mathrm{g}$

based on these criteria: two from the low volume group, one from the high-volume group, two from the low volume plus wrap group, and one from the high volume plus percussive wrap group.

Because of the limitations of the field of view for the SPECT system used in the ${ }^{99 \mathrm{~m}} \mathrm{Tc}-\mathrm{DTPA} / \mathrm{ASO}$ study, the lumbar spinal area could not be imaged and so the success of the injections could not be assessed as was done with the ${ }^{64} \mathrm{Cu}$-DOTA experiment. Instead, we used the tissue concentrations of the co-injected ASO in the lumbar spinal tissue to determine technical success of the IT injections. From the graph of the lumbar spinal cord ASO concentrations in Fig. 1b, it is quite apparent that two animals from the group injected with low volume without percussive wrap, two animals from low volume plus percussive wrap group, one animal from high volume without percussive wrap group and one animal from the high volume with percussive wrap group had very low tissue concentrations when compared to the other animals in these groups $(<3 \mu \mathrm{g} / \mathrm{g})$. These six animals were termed technical missed dose animals and removed from further analysis.

Increasing dose volume or adding external percussion increases rostral ${ }^{64} \mathrm{Cu}$-DOTA distribution along the neuraxis To determine if drug volume or percussion could alter distribution of an IT delivered drug, ${ }^{64} \mathrm{Cu}$-DOTA was injected IT into cynomolgus monkey. The compound was delivered under four different paradigms, low volume (0.4 $\mathrm{mL}, 3 \%$ of CSF volume) without percussion, high volume $(1.8 \mathrm{~mL}, 15 \%$ of CSF volume) without percussion, low volume with percussion, and high volume with percussion. These four paradigms were evaluated in three different experiments using the same 6 animals: the first evaluated low versus high injection volume, the second evaluated low volume versus low volume with percussion and the third evaluated high volume versus high volume with percussion.

Basic in-life tolerability assessments were included to confirm the tolerability of the manipulations, and that they did not alter vital signs that may contribute to CSF dynamics. The radiotracer, ${ }^{64} \mathrm{Cu}$-DOTA, was well-tolerated. Percussion treatments, delivered using a percussive wrap and HFCWO, were also well-tolerated. Vital signs were within normal ranges for cynomolgus monkeys: heart rate was $132 \pm 23 \mathrm{BPM}$, respiratory rate was $31 \pm 8$ respirations $/ \mathrm{min}$, and body temperature was $96.9 \pm 1.9^{\circ} \mathrm{F}$.

The AUC of the ${ }^{64} \mathrm{Cu}$-DOTA was observed in the lumbar spine near the injection site with the concentration decreasing along a caudal to rostral gradient. Comparing the low and high-volume IT injection conditions, there were no significant differences between the ${ }^{64} \mathrm{Cu}$-DOTA AUC values in the lumbar spinal cord, the site nearest to the injection, of the two volumes (Fig. 2a). The AUC values in the cranium and the thoracic spine were significantly higher in the high-volume condition than the 


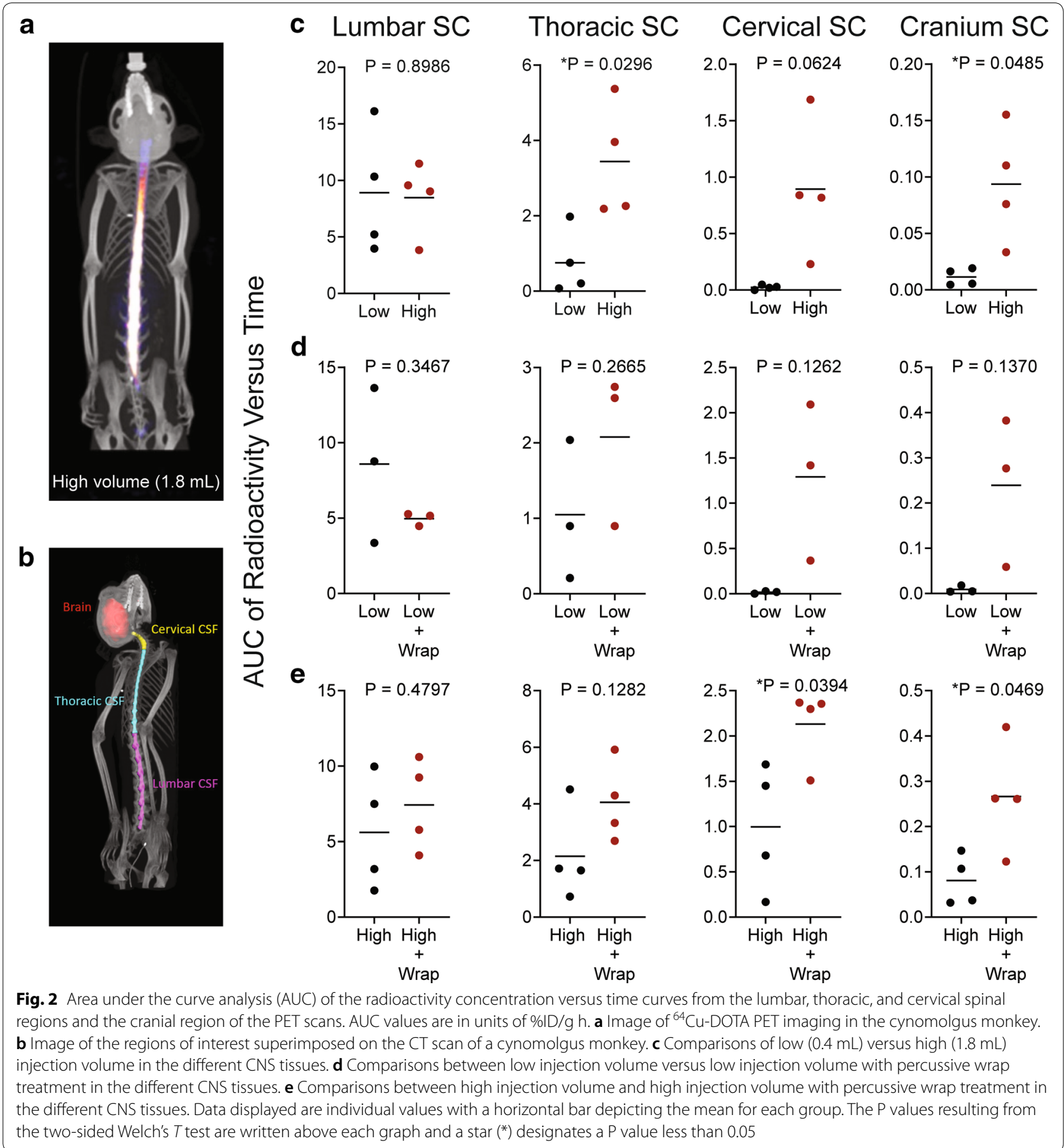

low volume condition. There was also a nonsignificant trend towards higher cervical spinal concentrations with the higher volume compared to the low volume injection $(\mathrm{P}=0.0624)$.

To determine if percussive wrap treatment could further influence distribution, we compared low and high volumes of ${ }^{64} \mathrm{Cu}$-DOTA with percussive wrap treatment (Fig. 2d, e respectively). With low volume IT injections, percussive wrap treatment resulted in a trend towards greater rostral distribution of the ${ }^{64} \mathrm{Cu}$-DOTA, with a clear trend for higher AUC values in the more rostral cervical spine and cranial regions with wrap treatment (Fig. 2d). With high-volume IT injections, percussive 
wrap treatment also resulted in greater rostral distribution of the ${ }^{64} \mathrm{Cu}$-DOTA, significantly increasing rostral distribution of the high volume ${ }^{64} \mathrm{Cu}$-DOTA to the cervical spine and cranial regions (Fig. 2e).

\section{Increasing dose volume or adding external percussion} to low dose volumes increases rostral ${ }^{99} \mathrm{~m}$ Tc-DTPA and ASO distribution along the neuraxis

To determine if we could replicate the results from the ${ }^{64} \mathrm{Cu}$-DOTA experiment with a therapeutically relevant modality, ASOs, and extend the findings to further understand the kinetics of distribution, we co-injected ASO with ${ }^{99 m}$ Tc-DTPA. There were again four different paradigms, low volume $(0.8 \mathrm{~mL}, 7 \%$ of CSF volume) without percussive wrap, high volume $(2.4 \mathrm{~mL}, 20 \%$ of CSF volume) without percussive wrap, low volume with percussive wrap, and high volume with percussive wrap. These different paradigms were tested in the same experiment using different groups of monkeys for each treatment.

After IT co-injecting ${ }^{99 \mathrm{~m}}$ Tc-DTPA with MALAT1 ASO the animals were imaged using SPECT at 0 min (prior to percussive wrap treatment), $40 \mathrm{~min}$ (after percussive wrap treatment) and $6 \mathrm{~h}$ post-injection. The images were limited to the cranial, cervical, and thoracic spinal cord due to limitations of the SPECT system's field of view. In the low injection volume with no percussive wrap group (Fig. 3a) the radioisotope progressed up the spinal column. Addition of percussive wrap treatment to the low dose volume (Fig. 3b) increased the rostral distribution of the radioisotope signal into the cervical spine and brainstem area. Increasing the dose volume from low to high with no percussive wrap treatment (Fig. 3c) resulted in greater rostral distribution in the base of the brain and into the transverse fissure between cerebellum and cerebrum and the lateral fissure between the temporal and frontal lobes. Addition of percussive wrap treatment to the high dose volume did not appreciably affect the distribution of the radioisotope over high volume alone (Fig. 3d).

To determine the distribution of ASO into tissues, samples from the lumbar, thoracic, cervical spinal cord, and frontal cortex were harvested 7 days after injection and analyzed for concentrations of ASO. Consistent with the SPECT imaging (Fig. 3), there was a nonsignificant trend towards increased concentrations of the ASO in the rostral neuraxis (frontal cortex) by increasing the dose volume (Fig. 4a, Table 2), whereas the percussive wrap treatment did not lead to increases in rostral distribution based on the tissue concentrations in either the low or high volume groups (Fig. 4b, c).

We have found that measurement of the pharmacological action of the ASO is a more sensitive assay for distribution of the ASO than measurement of the tissue concentrations. Therefore, tissue samples adjacent to those used for the ASO tissue concentration determinations were processed and analyzed for MALAT1 RNA expression by qRT-PCR. As the MALAT1 ASO is

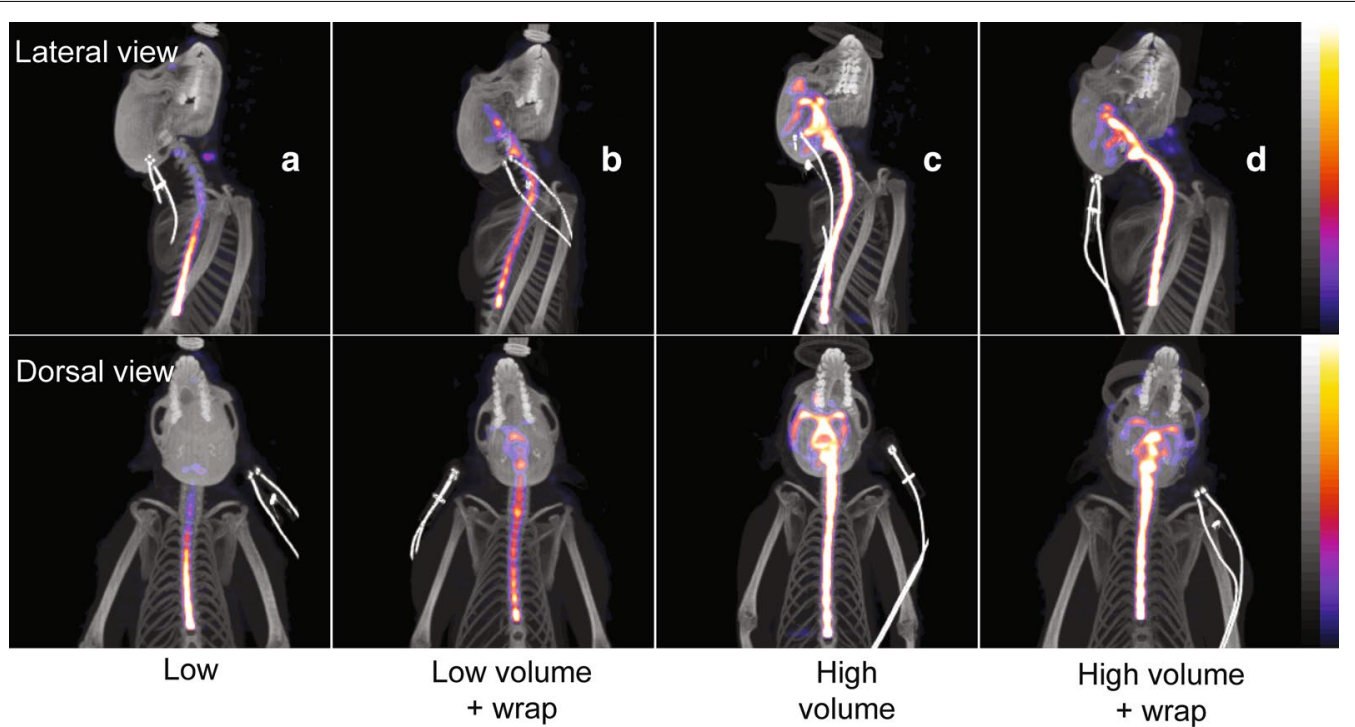

Fig. 3 Co-registered maximum intensity projection SPECT and CT images at 40-min post-injection from representative animals from the low $(0.8 \mathrm{~mL})$ volume $(\mathbf{a})$, low volume plus percussive wrap $(\mathbf{b})$, high $(2.4 \mathrm{~mL})$ volume $(\mathbf{c})$, or high volume plus percussive wrap $(\mathbf{d})$ treatment groups. The $99 \mathrm{~m}$ Tc-DTPA tracer incorporated into the dosing solution appears as bright areas within the spinal and cranial areas. SPECT images are scaled from 0 to $1 \%$ injected dose/g indicated by the color scale bar. Bright artefacts seen on the images are pulse oximeter sensors placed on the ear 


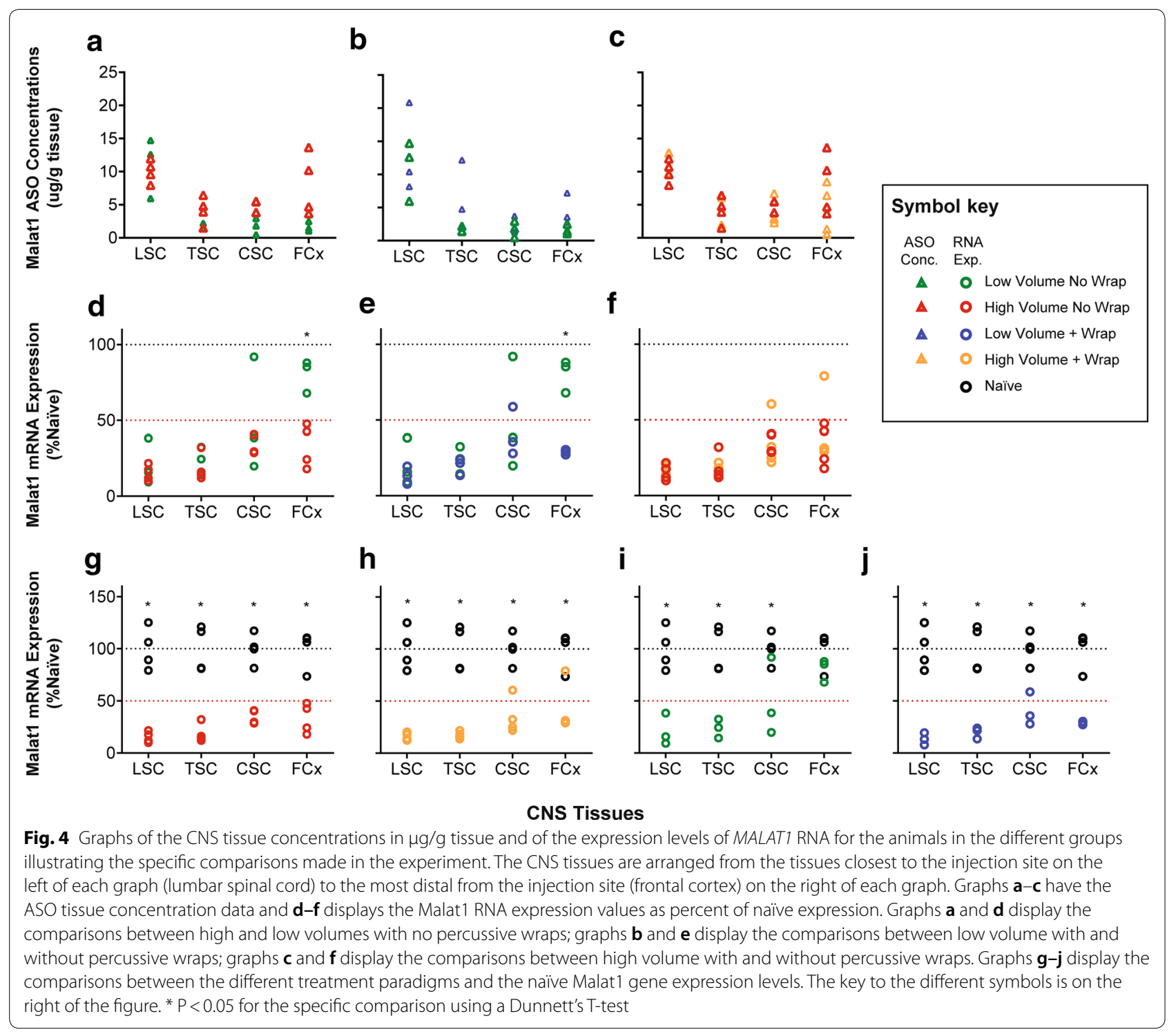

Table 2 P values for Tukey's multiple comparisons test for ASO concentration results

\begin{tabular}{lllll}
\hline Comparisons & \multicolumn{2}{l}{ Adjusted P value } & & \\
\cline { 2 - 5 } & Lumbar spinal cord & Thoracic spinal cord & Cervical spinal cord & Frontal cortex \\
\hline Low volume no wrap vs. high volume no wrap & 0.9818 & 0.8036 & 0.7176 & 0.1114 \\
Low volume no wrap vs. low volume + wrap & 0.8994 & 0.4168 & 0.9976 & 0.7696 \\
High volume no wrap vs. high volume + wrap & 0.1791 & 0.9998 & 0.9807 & 0.4357 \\
\hline
\end{tabular}

designed to suppress $M A L A T 1$ RNA, this is a measure of pharmacodynamic effect. MALAT1 RNA expression is significantly suppressed in the frontal cortex in the high volume no wrap group compared to the low volume no wrap group, suggesting that use of higher injection volumes provides greater rostral distribution of the ASO
(Fig. 4d, Table 3). Addition of percussive wrap treatment to the low injection volume also resulted in significant suppression of the MALAT1 RNA expression in the frontal cortex compared to the low volume with no wrap group (Fig. 4e, Table 3). This demonstrates that addition of percussive forces to low injection volumes 
Table 3 P values for Tukey's multiple comparisons test for Malat1 RNA expression results

\begin{tabular}{|c|c|c|c|c|}
\hline \multirow[t]{2}{*}{ Comparison } & \multicolumn{4}{|l|}{ Adjusted P value } \\
\hline & Lumbar spinal cord & Thoracic spinal cord & Cervical spinal cord & Frontal cortex \\
\hline Naive vs. low volume no wrap & $<0.0001^{*}$ & $<0.0001^{*}$ & $0.0008^{*}$ & 0.463 \\
\hline Naive vs. low volume + wrap & $<0.0001^{*}$ & $<0.0001^{*}$ & $<0.0001^{*}$ & $<0.0001^{*}$ \\
\hline Naive vs. high volume no wrap & $<0.0001^{*}$ & $<0.0001^{*}$ & $<0.0001^{*}$ & $<0.0001^{*}$ \\
\hline Naive vs. high volume + wrap & $<0.0001^{*}$ & $<0.0001^{*}$ & $<0.0001^{*}$ & $<0.0001^{*}$ \\
\hline Low volume no wrap vs. high volume no wrap & 0.9877 & 0.9916 & 0.6978 & $0.0018^{*}$ \\
\hline Low volume no wrap vs. low volume + wrap & 0.9731 & 0.9976 & 0.9477 & $0.0014^{*}$ \\
\hline High volume no wrap vs. high volume + wrap & $>0.9999$ & $>0.9999$ & $>0.9999$ & 0.9086 \\
\hline
\end{tabular}

*indicates a $\mathrm{P}<0.05$

can increase the rostral distribution of the ASO over low injection volume alone. When the compressive wrap was added to the high injection volume there was no further suppression of MALAT1 RNA expression over high volume alone, suggesting that addition of compressive forces to the high dosing volume does not further improve rostral distribution (Fig. 4f, Table 3). When the MALAT1 RNA expression with the different treatment paradigms was compared to the naïve animal expression levels, significant suppression of RNA expression was observed in every comparison (Fig. $4 \mathrm{~g}-\mathrm{j}$ ) except in the frontal cortex with the low volume no percussive wrap treatment (Fig. 4i). Taken together, either increasing dose volume, or adding percussive wrap treatment to the low injection volume, could increase rostral distribution of ASOs.

To further confirm ASO distribution and pharmacology on a cellular level, spinal cord and frontal cortex histological samples were stained for MALAT1 RNA via in situ hybridization (ISH) and for ASO via immunohistochemistry (IHC) (Fig. 5). The IHC staining for the ASO clearly demonstrates good distribution of the ASO throughout the CNS in the high volume no wrap treated animal and lesser distribution to rostral structures more distal to the injection site in the low volume no wrap treated animal. The MALAT1 ISH demonstrates complete reduction of RNA expression in all the CNS regions evaluated in the animal from the high volume treated group. In contrast, the MALAT1 ISH images from the low volume group animal shows complete reduction of RNA expression in the spinal cord regions and only partial reduction of MALAT1 RNA expression in the frontal cortex. These results are consistent with the ASO concentration, PCR, and imaging data. Naïve tissues were stained for ASO IHC but there was no staining of any structure in the CNS so these images have been omitted from Fig. 5 for brevity.

\section{Broad CNS pharmacology following high volume delivery of a MAPT-ASO}

Suppression of human MAPT has been proposed as a potential therapy for tauopathies, including Alzheimer's disease and frontotemporal dementia [8]. Indeed, ASOs suppressing Mapt in rodent models of disease can reverse pathology and ameliorate phenotype [8]. Since many of the potential indications for a MAPT ASO likely require cortical suppression, we evaluated whether we could increase distribution and pharmacology of a MAPT ASO that targets monkey MAPT mRNA by using a high volume $(2 \mathrm{~mL})$ compared to a low dose volume $(0.8 \mathrm{~mL})$. We found a trend toward increased ASO concentrations in the more rostral structures in the high-volume group compared to the low volume group (Fig. 6a). Consistent with the ASO concentration data, increasing the dose volume significantly increased the pharmacological action of the ASO, with greater reduction in MAPT mRNA in the high-volume group compared to the low volume group (Fig. 6b). Taken together, using a therapeutically relevant modality, increasing the convective forces within the CSF following an IT drug delivery can increase ASO concentration and pharmacological action throughout the CNS.

\section{Discussion}

We embarked on this series of experiments to evaluate how manipulation of convective forces affected the rostral distribution of drug after lumbar intrathecal administration in the monkey. The objective of this work is to incorporate the lessons learned here in clinical practice to potentially increase the cranial distribution of IT delivered therapeutics. Our earlier work in rodents demonstrated that adjusting the volume of injection can increase neuraxial exposure distal to the lumbar IT injection site [7]. There is a small body of literature that describes the effects of several convective mechanisms in the CNS space on CSF motion including CSF turnover, cardiac motion, respiratory thoracic motion, and body 


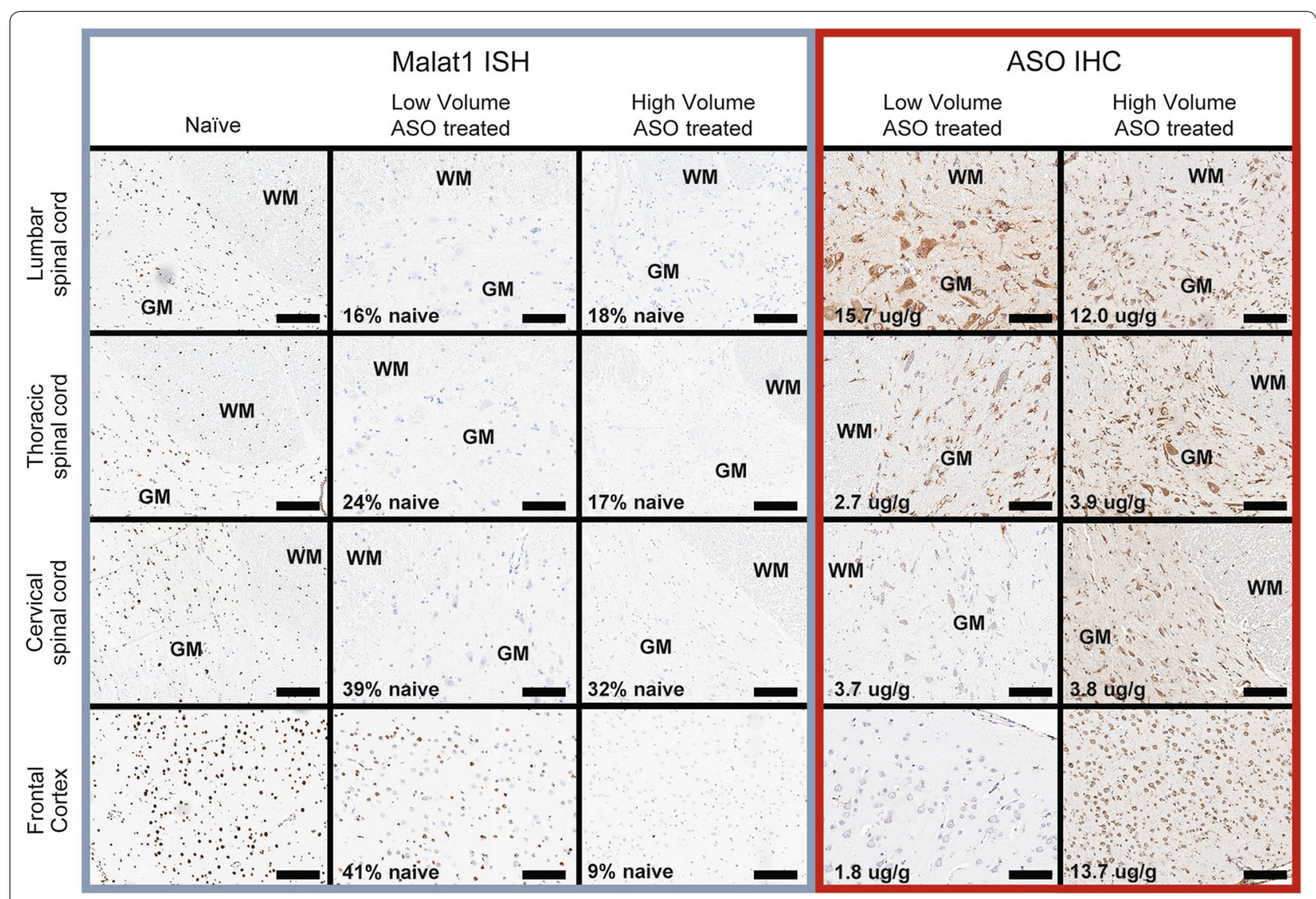

Fig. 5 Imaging the spinal cord and frontal cortex ASO distribution and pharmacology. Representative images from MALAT1 ISH (left three columns) and ASO IHC (two columns on the right) in representative animals from the naïve animal, the low $(0.8 \mathrm{~mL})$ volume no wrap, and high (2.4 $\mathrm{mL})$ volume no wrap groups. Images are arranged top to bottom with the proximal (lumber spinal cord) to distal (frontal cortex) areas from the injection site. In the spinal cord pictures, white matter is designated by WM and gray matter by GM. The tissue concentrations in ug/g tissue measured by LC-MS in the adjacent tissues are displayed in the ASO IHC pictures. The MALAT1 RNA expression values (percent of naïve control) as measured by PCR from the adjacent spinal cord tissues are written on the ASO treated MALAT1 ISH pictures. Horizontal black bars are scale bars representative of $100 \mu \mathrm{m}$

movement [3-6]. We chose injection volume and exogenous forces applied with a percussive wrap as potential modifiers of ASO distribution, as these would be easily incorporated into the clinical environment.

Although increasing dose volume has not been systematically taken advantage of in IT drug therapy, injected volumes of up to $50 \mathrm{~mL}$ (approximately $33 \%$ of the total CSF volume) have previously been given safely in humans. Moreover, myelography contrast injection guidelines call for up to $17 \mathrm{~mL}$ IT injection of CT contrast media in adults $[9,10]$.

In our first experiment, ${ }^{64} \mathrm{Cu}$-DOTA was successfully injected IT and imaged in cynomolgus monkeys in high $(1.8 \mathrm{~mL})$ or low $(0.4 \mathrm{~mL})$ bolus volumes, with or without exogenous force applied to the thorax via a percussive wrap. There was an increase of rostral radioactivity distribution distal to the injection site with the larger bolus dose volume compared to the lower dose volume. Rostral radioactivity distribution was also increased with the addition of the percussive wrap in both the low and high dosing volumes.

These results then led to our next experiment dosing a pharmacological dose of an unlabeled ASO to the nonprotein coding RNA MALAT1 spiked with ${ }^{99 \mathrm{~m}} \mathrm{Tc}-\mathrm{DTPA}$ to bridge the previous radioactive imaging results with ASO distribution using dose volume and percussive wrap. We examined distribution of the radioactive tracer, ASO concentrations and the reduction of the expression of the MALAT1 RNA which is the pharmacological effect of the ASO and a much more sensitive assay for the presence of the ASO than bioanalysis of the molecule itself. We again found that the larger injection volume increased rostral distribution of the radioactivity and the MALAT1 ASO and decreased the RNA expression of MALAT1 distal from the lumbar injection site. We also found that percussive wrap treatment increased the rostral distribution 


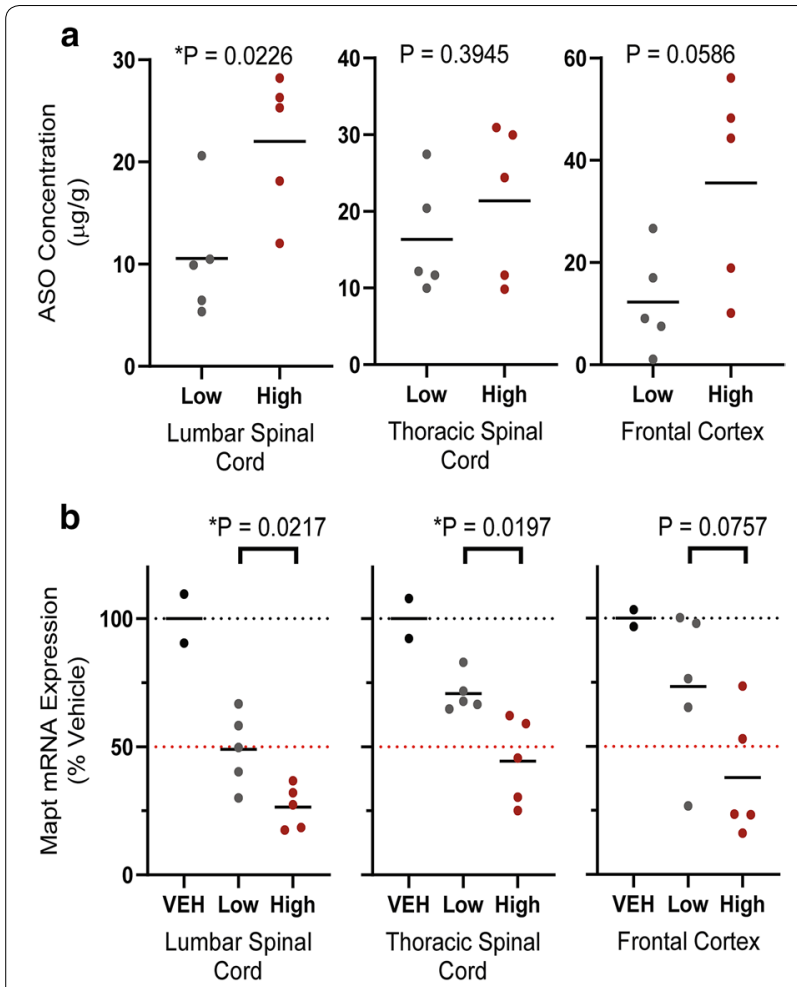

Fig. 6 Effect of injection volume on the CNS distribution and pharmacology of an ASO specific to the MAPT gene. a Graphs of the tissue concentrations ( $\mu \mathrm{g} / \mathrm{g}$ tissue) of the MAPT ASO in the lumbar and thoracic spinal cord, and frontal cortex for the animals treated with low $(0.8 \mathrm{~mL}$, gray) or high ( $2.0 \mathrm{~mL}$, red) dose volume. b Graphs for the MAPT mRNA expression for the low (gray) versus high (red) volume treatments as a percentage of the vehicle treated (black) animals. At the top of each graph is the $P$ value for the comparison of the low versus high volume treatment as calculated with two tailed Welch's T-test. A star $\left(^{*}\right)$ designates a P value less than 0.05 . Data is depicted as individual values with horizontal bar depicting the mean value for the group

of the radioactivity and MALAT1 ASO, as evidenced by the target RNA expression reduction, above that seen with the low dose volume. However, percussive wrap did not seem to increase rostral distribution of the ASO, as evidenced by the decrease in MALAT1 RNA expression, or increase radioactivity above that seen with the high dose volume.

We then utilized the lessons learned from these two experiments and evaluated the distribution of an ASO against MAPT after lumbar intrathecal administration dosed in a low $(0.8 \mathrm{~mL})$ or high $(2 \mathrm{~mL})$ volume. We did not adopt the compressive wrap in this experiment since the evidence from the previous experiments suggested that similar rostral distribution of ASOs can be achieved by changing the injection volume alone. This intervention is also more adoptable by clinicians to enhance the rostral distribution of ASOs. We again found an increase in rostral distribution of the MAPT ASO distal from the lumbar injection site with the larger volume when compared to the smaller volume. The rostral distribution of the MAPT ASO to the cranium is important because this target is important in frontotemporal dementia and Alzheimer's disease, both neurodegenerative diseases of the brain. These convective forces effects were also evident in the increase in the pharmacological action of the ASO in the high dose volume animals compared to the low dose volume ones.

It is likely that both increased dose volume and mechanical percussion involve convection enhancement. In addition to increasing radioactive tracer concentrations in the more cranial regions of the neuraxis, the percussive wrap reduced tracer concentration in the lumbar region in the low volume condition. The use of increased dose volume and exogenous mechanical force may thus be useful for increasing IT therapeutic dose to cranial regions and reducing exposure at the lumbar site of delivery when required. Conversely, for IT dosed drugs targeted for local lumbar spinal cord or nerve root action where neuraxial spread is not desired, it may be useful to minimize convective force by using lower dose volumes.

We evaluated the CSF volume in a large group of cynomolgus monkeys using T2-weighted volumetric MRI methods. We found that the cynomolgus monkey has a total CSF volume of $11.9 \pm 1.6 \mathrm{~mL}$ (mean \pm standard deviation). This value was used to guide selection of IT bolus injection volumes to model published high and low intrathecal injection volumes delivered in the clinic.

Total CSF volume in humans is generally quoted as approximately $130 \mathrm{~mL}$ [12]. This number is derived from Dixon and Halliburton in 1916 [13] that stated the total CSF volume in humans to be $100-130 \mathrm{~mL}$ based on the "estimations of several observers". Recently, Chazen et al. [14] published a paper using T2-weighted volumetric MRI methods in the human similar to what we employed here. They found a volume of $263 \pm 55 \mathrm{~mL}$ (mean \pm standard deviation) in a group of 15 normal human subjects, which is much larger than the traditional $130 \mathrm{~mL}$ volume. When we re-evaluate our injection volume percentages of total CSF volume using these new human data, they change from $3-7 \%$ to $1.4-3 \%$ for the low volume doses and from $15-20 \%$ to $7-10 \%$ for the high-volume doses. When allometrically scaling intrathecal dosing between monkey to human, what was previously a $130 / 11.9 \mathrm{~mL}$, or an approximately tenfold higher CSF volume in the human, now becomes a 263/11.9 $\mathrm{mL}$ or approximately 20 -fold higher CSF volume in the human than in the monkey.

Clear assessment of the variability in the quality of IT radiotracer delivery by injection as revealed by imaging also illustrates the value of molecular imaging in 
assessing efficiency of IT therapeutics delivery. IT nuclear imaging, which has long been used to evaluate CSF leaks, may provide great value in understanding the efficiency and pharmacodynamics of IT dosed therapeutics. Experiments in humans are required to assess the translatability of the volume principles demonstrated by the experiments within this manuscript. The assumption that rostral distribution as a function of percentage of CSF volume injected is consistent from NHP to human has yet to be tested. Nuclear imaging will allow us to test these volume principles in humans. It may also be crucial in determining differences in intrathecal dynamics in different disease states, for instance where cerebral ventricular volumes are increased, or where cortical atrophy increased subarachnoid CSF space.

\section{Conclusions}

Recently, therapeutic molecules that do not cross the blood brain barrier, such as peptides, proteins and ASOs, have been evaluated for treating diseases and conditions of the CNS. The most obvious clinical route of delivery for these novel CNS therapeutics is delivery into the CSF by lumbar puncture IT administration. We have found that convective forces during IT dosing by lumbar puncture are important for delivering the therapeutic to the rostral structures of the neuraxis. Our results suggest that increasing the volume of the dose can significantly increase rostral distribution of PET and SPECT imaging agents, as well as therapeutic ASOs. With smaller injection volumes, the addition of exogenous convective forces by treatment with percussive wraps can improve the rostral distribution of the ASOs to the level of the higher dose volume, but do not improve rostral distribution over the high dose volume. We feel that clinically the adoption of high dose volume is appropriate for therapeutic molecules for CNS diseases and conditions where the target area is in the rostral CNS structures.

\footnotetext{
Abbreviations

IT: Intrathecal; CNS: Central nervous system; CSF: Cerebral spinal fluid; ASO: Antisense oligonucleotide; NHP: Nonhuman primate; MRI: Magnetic Resonance Imaging; PET: Positron emission tomography; SPECT: Single Photon Emission Computed Tomography; CT: Computed tomography; $\mathrm{Cm}^{3}$ : Cubic centimeters; BBB: Blood brain barrier; DOTA: Dodecane tetraacetic acid; DTPA Diethylenetriaminepentaacetic acid; MAPT: Microtubule associated protein tau; NBR: Northern Biomedical; Malat1: Metastasis associated lung adenocarcinoma; SC: Subcutaneous; IM: Intramuscular; FSE: Fast spin echo; ROI: Region of interest; HFCWO: High-frequency chest wall oscillation; ITLC: Instant thin layer chromatography; OSEM2D: Ordered Subset Expectation Maximization; \%ID/g: Percent injected dose per gram; MOE: 2' Methoxyethyl; aCSF: Artificial cerebral spinal fluid; MLEM: Maximum likelihood estimation method; QF: Quantification factor; MIP: Maximum intensity projection; GRT-PCR: Quantitative reverse transcriptase polymerase chain reaction; LC-MS/MS: Liquid chromatograph with mass spectrometry detection; TEA: Triethyalamine; HFIP: Hexo-fluoroisopropanol; IS: Internal standard; ACD: Advanced Cell Diagnostics; DAB: 3,3'-Diaminobenzidine chromogen; AUC: Area under the curve; ISH: In situ
}

hybridization; IHC: Immunohistochemistry; CV\%: Percent coefficient of variation; SD: Standard deviation.

\section{Acknowledgements}

The authors would like to acknowledge the scientists, staff, and technicians at MPI Research and Invicro for their precise execution of the studies described in this paper. The authors would like to specifically thank Kevin Magalhaes, Joshua Higgins, and Carolynn Gaut for their hard work and dedication to the project management of the imaging studies and Howard Dobson for useful discussions and his valuable insight. The authors would also like to acknowledge Tracy Reigle and Wanda Sullivan for their assistance with the figures in this publication.

\section{Authors' contributions}

JMS and CM assisted in designing the experiments and analyzed data and are co-primary authors of the manuscript. CM also conducted experiments and collected data. BP, LT, BD-S and BF conducted experiments, collected, analyzed data and assisted in writing the manuscript. RP and JH analyzed data and assisted in writing the manuscript. DAW and LH assisted in designing experiments and writing the manuscript. SH assisted in designing and conducting the experiments and writing the manuscript. NC and HK assisted in writing the manuscript and interpreting the data. AV and JH assisted in designing experiments, analyzed data and assisted in writing the manuscript. EES assisted in designing the experiments and writing the manuscript and is the senior member of the lonis group. All authors read and approved the final manuscript.

\section{Funding}

The research described herein was wholly funded by Biogen and lonis Pharmaceuticals, Inc.

\section{Availability of data and materials}

The datasets used and/or analyzed during the current study are available from the corresponding author on reasonable request.

\section{Ethics approval and consent to participate}

The MRI quantification of CSF volume experiment in the monkey, ${ }^{64} \mathrm{Cu}$-DOTA and ${ }^{99 \mathrm{~m} T c-D T P A} /$ Malat1 ASO experiments were performed at MPI Research and the study procedures were reviewed and approved by the Institutional Animal Care and Use Committee at MPI Research prior to the start of the study. The ${ }^{64} \mathrm{Cu}$-DOTA study was performed under approved protocols 1881 091 and 1881-100. The ${ }^{99 \mathrm{~m} T C-D T P A / M a l a t 1 ~ A S O ~ e x p e r i m e n t ~ w a s ~ p e r f o r m e d ~}$ under approved protocol 1881-102 and the MRI experiment was performed under protocols 1881-098 and 1881-100. The Mapt ASO experiment was performed at Northern Biomedical Research under a protocol approved by their Institutional Animal Care and Use Committee, specifically protocol number 004-031. All three of these studies complied with the U.S. Department of Agriculture's Animal Welfare Act (9 CFR Parts 1, 2, and 3) and the Guide for the Care and Use of Laboratory Animals, Institute of Laboratory Animal Resources, National Academy Press, Washington, D.C., 2011. Both MPI Research and Northern Biomedical Research are accredited by the Association for Assessment and Accreditation of Laboratory Animal Care (AAALAC).

\section{Consent for publication \\ Not applicable.}

\section{Competing interests}

CM., B.F., B.P., L.T., B.D-S., H.K. and E.S. are employees of and stockholders in Ionis Pharmaceuticals, Inc. J.H. is a co-founder and managing partner of Invicro as well as an equity shareholder. J.H., J.M.S., and R.P. were employees of Invicro at the time that this work was performed, and J.M.S. is currently an employee of Biogen Inc., L.H. was an employee of Biogen at the time that this work was performed and is currently an employee of Kaiser Permanente, D.A.W, and N.C. are employees of Biogen. A.V. was an employee of Biogen at the time that this work was performed, and is currently an employee of Codiak Pharmaceuticals, Inc.

\footnotetext{
Author details

${ }^{1}$ Ionis Pharmaceuticals, Inc., 2855 Gazelle Court, Carlsbad, CA 92010, USA.

${ }^{2}$ Biogen, Inc., Cambridge, MA, USA. ${ }^{3}$ Invicro, LLC, Boston, MA, USA. ${ }^{4} \mathrm{MPI}$
}

Research, Mattawan, MI, USA. 
Received: 27 March 2020 Accepted: 28 July 2020

Published online: 08 August 2020

\section{References}

1. Mercuri E, Darras BT, Chiriboga CA, Day JW, Campbell C, Connolly AM, et al. Nusinersen versus sham control in later-onset spinal muscular atrophy. N Engl J Med. 2018;378(7):625-35.

2. Hocking G, Wildsmith JA. Intrathecal drug spread. Br J Anaesth. 2004;93(4):568-78.

3. Hsu Y, Hettiarachchi HD, Zhu DC, Linninger AA. The frequency and magnitude of cerebrospinal fluid pulsations influence intrathecal drug distribution: key factors for interpatient variability. Anesth Analg. 2012;115(2):386-94.

4. Hettiarachchi HD, Hsu Y, Harris TJ Jr, Penn R, Linninger AA. The effect of pulsatile flow on intrathecal drug delivery in the spinal canal. Ann Biomed Eng. 2011;39(10):2592-602.

5. Hladky SB, Barrand MA. Mechanisms of fluid movement into, through and out of the brain: evaluation of the evidence. Fluids Barriers CNS. 2014;11(1):26

6. Brinker T, Stopa E, Morrison J, Klinge P. A new look at cerebrospinal fluid circulation. Fluids Barriers CNS. 2014;11:10.

7. Wolf DA, Hesterman JY, Sullivan JM, Orcutt KD, Silva MD, Lobo M, et al. Dynamic dual-isotope molecular imaging elucidates principles for optimizing intrathecal drug delivery. JCI Insight. 2016;1(2):e85311.
8. DeVos SL, Miller RL, Schoch KM, Holmes BB, Kebodeaux CS, Wegener AJ, et al. Tau reduction prevents neuronal loss and reverses pathological tau deposition and seeding in mice with tauopathy. Sci Transl Med. 2017;9(374):eaag0481.

9. Gold LH, Leach CG, Kieffer SA, Chou SN, Peterson HO. Large-volume myelography. An aid in the evaluation of curvatures of the spine. Radiology. 1970;97(3):531-6.

10. Kieffer SA, Binet EF, Esquerra JV, Hantman RP, Gross CE. Contrast agents for myelography: clinical and radiological evaluation of amipaque and pantopaque. Radiology. 1978;129(3):695-705.

11. Rieselbach RE, Di Chiro G, Freireich EJ, Rall DP. Subarachnoid distribution of drugs after lumbar injection. N Engl J Med. 1962;267:1273-8.

12. Yaksh TL. Spinal drug delivery. 1st ed. Amsterdam: Elsevier; 1999. p. 614.

13. Dixon WE, Halliburton WD. The cerebro-spinal fluid: IV. Circulation. J Physiol. 1916;50(3):198-216.

14. Chazen JL, Dyke JP, Holt RW, Horky L, Pauplis RA, Hesterman JY, et al. Automated segmentation of MR imaging to determine normative central nervous system cerebrospinal fluid volumes in healthy volunteers. Clin Imaging. 2017:43:132-5.

\section{Publisher's Note}

Springer Nature remains neutral with regard to jurisdictional claims in published maps and institutional affiliations.
Ready to submit your research? Choose BMC and benefit from:

- fast, convenient online submission

- thorough peer review by experienced researchers in your field

- rapid publication on acceptance

- support for research data, including large and complex data types

- gold Open Access which fosters wider collaboration and increased citations

- maximum visibility for your research: over $100 \mathrm{M}$ website views per year

At BMC, research is always in progress.

Learn more biomedcentral.com/submissions 